\title{
Industrial Cyber Physical System (ICPS) for the Control and Monitoring of Machines
}

\author{
${ }^{1}$ Abdulhaq Abildtrup and ${ }^{2}$ Iben Charlotte Alminde \\ ${ }^{1,2}$ Department of People and Technology, Roskilde University, Roskilde, Denmark \\ 1abdulhaqabildtrup@outlook.com
}

\begin{abstract}
ArticleInfo
Journal of Machine and Computing (http://anapub.co.ke/journals/jmc/jmc.html)

Doi : https://doi.org/10.53759/7669/jmc202101008

Received 20 December 2020; Revised form 26 January 2021; Accepted 25 March 2021; Available online 05 April 2021. (C)2021 The Authors. Published by AnaPub Publications.

This is an open access article under the CC BY-NC-ND license. (http://creativecommons.org/licenses/by-nc-nd/4.0/)

Abstract - The Industrial Cyber Physical System (ICPS) for industrial monitoring applying wireless technology i.e. Wireless Sensor Networks (WSNs). WSNs are a critical concern for engineering firms. There are various techniques incorporated in wireless networks for industrial application and machining in the modern age. In this research contribution, security protocols have been considered through the incorporation of novel design techniques for wireless industrial controlling and monitoring systems and eco-friendly machines. The wireless communication approaches are restricted to modest application due to their slow communication velocity, information security and communication distance. Moreover, they are influence by bad weather and noise conditions e.g. rain, fog and snow. This paper presents a proposal of a system for controls and monitoring of applications in engineering firms using Internet of Everything (IoE) and GPRS enhanced communication, high security measures and low costs without requiring infrastructural modification in the hardware of GSM operating segments.
\end{abstract}

Keyword - Industrial Cyber Physical System (ICPS), Internet of Everything (IoE), Wireless Sensor Networks (WSNs)

\section{INTRODUCTION}

Industrial control and monitoring is fundamental in the process of gathering the essential data, statistics and information about different industrial procedures, devices, eco-friendly machines and motors employed in engineering firms [1]. The major aim of controlled accessibility is to ensure high quality and effective productivity of industrial products being processed. Presently, remote control, and novel technological advancements such as RF, ZigBee and Infrared technologies have been utilized in firms. Over the past few decades, Wireless Sensor Networks (WSNs) have become considerably spread in the processing and transfer of industrial data and control applications. Technological enhancements have allowed classical and automated systems to be in place.

Apart from that, the present of fast processing of stabilized and more sensitive engineering products is of significant merit in industrial automation. Because of developments in initiatives of communication of Internet of Everything (IoE), schemes are not monitored or controlled completed in the modern age. However, systems are now automated using remoteaided or computer-assisted devices. Industrial environmental settings have been advancing everyday with the newly introduced automatic techniques due to eliminating conventional processes of manufacturing hence advancing more workload. The upcoming generation of industries will definitely be more automatic and advanced than to the present ones. This has brought about novel technologies of "smart companies" in this era of monitoring and control of different industrial applications.

Considered as an emerging technology due to rapid advancements of modern WSNs, IoE has gained popularity and is projected to bring about numerous applications in firms [2]. Recently introduced idea of IoE is giving an aided hand to attain industrial automations via remote access. In IoE, devices constituting systems will be capable of communicating and linking up with other devices or systems in the same locality over the same platform. As such, this stimulates the exchange of critical logs, statistics and data, including various parameters and information among different devices to enhance their performances, and this will aid industries to enhance their management, productivity and enhance throughput.

Flexibility is present in the reception segment. WSNs are robust over hazards such as fires or earthquakes. These utilize low power for battery application. The networks do not require special licenses or permissions required for use, which is utilized for the transmission of sensor information, and utilizes customized wireless transmission protocols that are designed based on reliability and simplicity. In this paper, we involve data broadcast algorithm for 802.15.4 centered ZigBee network protocol and their application. This research proposes the Zig-Bee transit and accepts information between microcontrollers and computers. Zig-Bee is applied to control speed and direction of DC monitor via dual h-bridge converters. A single Zig-Bee has been linked to individual computer that is utilized to accept and transfer information. ZigBee is linking the external devices via the serial port through the RS-232 standards. 
For face detection [3], users are capable of controlling or monitoring the complete wireless network using computer and visual centered GUI, which is meant for detecting and operating faces. This measure is utilized for security checks in the monitoring segment. The color of the skin is a critical cue for determining the faces of humans. Skin color distribution and cluster in minor regions is in the chromatic spaces of colors. Processing colors is more compared to processing of different facial features. To attain better form of accuracy, data on faces has to be utilized and two-dimensional and threedimensional faces evaluated critically. In that regard, the detection of the color of the skin is firstly performed on the input of color images to minimize the complexities of computation. Due to the skin color accuracy and the detection effect, the result of face detection scheme, selecting the best color spaces for skin color detection is fundamental. The general corrective algorithm is applicable to the problem of face detection

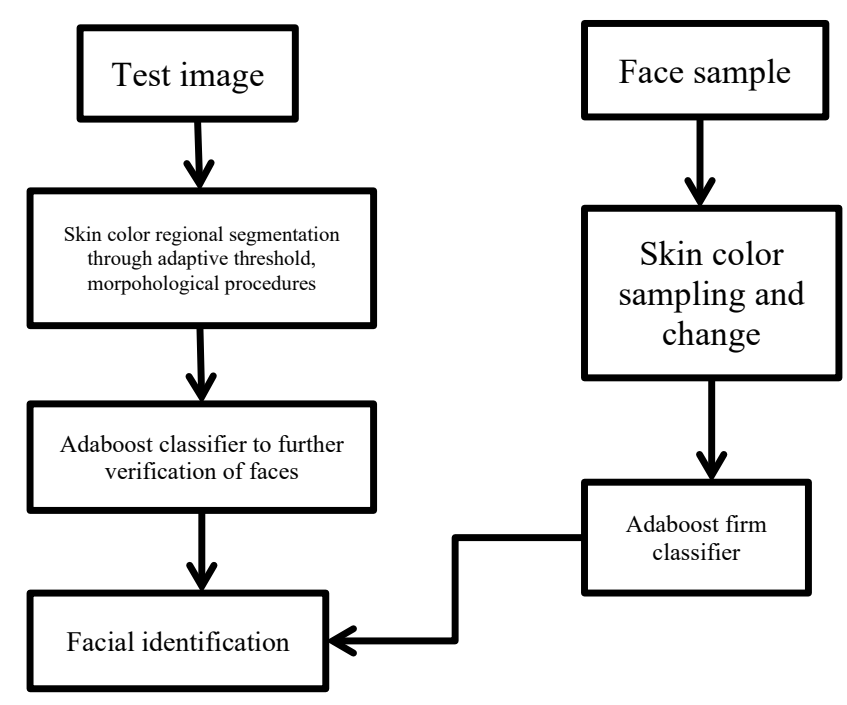

Fig 1: Face identification procedure

Automations are fundamental for the control of eco-friendly machines and procedure, which significantly minimize the necessity for human interference [4]. Because of prompts advancements of technology over the past few decades, the tracking system that checks the status of automated machines is what industries need at the moment and the system is utilized in different ways to display and track machine data and condition in actual time in devices and technologies such as GPRS, GSM and Zig-Bee. Presently, the systems are not completely automated; these have to be tracked moment to moment. Now, SCADA like systems are utilized for the purpose of automation; however, the issue is that the systems cannot be controlled from a completely remote locality.

In the industrial environment, some procedures are automated e.g., the satellite sector is initiative productions for the cables of optic fiber. In these procedures, parameters such as production attained, gas leakage, pressure etc., have to be controlled in actual-time from remote locality [5]. There are less skilled individuals in the industrial sector; so they have to be careful when evaluating moment parameters such as gas leakages, pressure and temperature and production. By considering this as a system of automation that is structured in a manner that in the absence of the concerned individual, updates and control measures of a particular plant will aid in the communication of GSM.

Various sensors are incorporated to retrieve data from the plant ecosystem. The sensor signal is provided to ARM controllers for conditioning of the signal conditioning and based on the need; controllers are programed and generate control signals meant to control operations. In the system, two or more tasks have to be monitored and controlled at the same moment. In these systems, uC/OS-II-RTOS is applied to effectively manage the allocation of resources to users in controlled or orderly manner by permitting the assignment of priority conversion.

In remote location, the system permits users to effectively control and monitor the office/house equipment and appliances through mobile devices that have been set by communicating commands in the form of SMSs and accepting the status of appliances. The major concept governing the project is accepting sent SMSs and processes meant to underpin particular operations. The form of operation is done and depends on the condition of the SMSs that have been sent. The rule in which the project is centered on is modest. The communicated SMSs is polled and stored in the receiver's mobile station and required control signals are generated and communicated to intermediate hardware, which have been structured based on the commands accepted as sent SMSs.

The part (Section I) has introduced the concept of industrial monitoring and control. From this introductory paragraph, it is seen that monitoring and control are utilized in gathering fundamental information, statistics and data about various industrial devices, procedures and machines, which are eco-friendly It is seen that the emerging technological advancement has WSNs applied widely in the engineering sector. The initiative is advantageous due to its flexibility and minimal effects which are robust compared to the examples given in this section i.e. earthquakes and fires. WSNs have also been seen to be essential since they utilize low power to the optimization of batteries. 
The remaining section will provide an in-depth evaluation of the WSNs concept in the establishment of a system design, i.e. Industrial Cyber Physical System (ICPS), for monitoring and control of machines. The remaining section has been arranged as follows: Section II presents a background analysis of the research. Section III reviews the relevant literature regarding Industrial Cyber Physical System (ICPS) for the Control and Monitoring of Machines. Section IV presents the proposed system architecture. Finally, Section V concludes the paper and present future directions.

\section{BACKGROUND ANALYSIS}

In this research contribution, Industrial Cyber Physical System (ICPS) has been proposed to monitor and control applications in engineering firms using IoE and GPRS-based communication. It system is proposed and effective due to its affordability and safety and necessitates minimal infrastructural change in hardware and GSM. In this paper, incorporates security protocols and rules through the incorporate of new design methods that apply WSNs in the control and monitoring of eco-friendly machines. WSNs methods are constrained to modest application since they are slow in speed, data privacy is guaranteed and the distance is wider i.e. longevity.

ICPS will provide researchers with a basis to conduct further research on how to enhance monitoring and control using IoE. The system integrates physical firms with cyber networks and structural systems hence make them to operate harmoniously and gain merits from integrated designs and ranged optimization. The safety and performance of systems can be developed through structural data infrastructures and methods of control and monitoring. This is projected to uphold controllability with reference to external disturbances and unplanned faults.

This paper investigates the present status of ICPS where similarities and contrasts between CPS and ICPS are done. The analogue practices of industrial application have seen to provide real-time monitoring and control issues. Wireless communication is another key concept evaluated in this research as a major factor considered in the establishment of the proposed system. According to the research, wireless communication concept represents the transfer of data through various distances without essentially applying wireless conductors and electrical wires.

In this research, we have utilized and analyzed:

- Zig-Bee network for industrial information communication and its rule and features evaluated

- Collision Elimination

- GSM Networks

- System's Hardware

The system control and monitoring is attained in industrial application and particular sub-factors considered in the designing of the system:

- $\quad$ Micro-Controllers

- Current Sensors

- Temperature Sensors

- GSM Modules.

For GSM modules, industrial applications and parameters more than its projected threshold figure can be controlled through micro-controllers based on TC-35-I GSM. As for the cellular application, in an aim to make them operate effectively, it is fundamental that the modules are securely connected to the host. The section below evaluates the relevant literature assumption regarding the application of WSNs in the designing of the proposed system.

\section{LITRETURE REVIEW}

A. Ogden-Swift in [6] argues that various control technologies are utilized for control and monitoring of Industrial Cyber Physical System (ICPS), whereby communication between systems and users is particularly realized online through wireless communication methods e.g. Bluetooth, Zig-Bee and RF. Moreover, SCADA programs are used for the development of the interface for users. Nonetheless, the programs do not assure adaptability for the users due to their costly libraries. Bluetooth, Zig-Bee and RF technologies have been considered in easily applicable applications because of the short range evident between the users' receiver and sender incorporates minor data volumes being transferred.

X. Xiaoli and W. Huan in [7] comment that Bluetooth, Zig-Bee and RF wireless methods are restricted to modest applications due to their slower communication velocity, distance and information security. Presently, time-based schemes have been incorporated with remote-assisted systems because of advancement of technology. In these schemes, it is considered as a critical concern to retrieve data about controls; however, the conditions of eco-friendly devices and machines can be determined through the internet.

A. Çakir, H. Çalis and G. Turan in [8] have done an analysis on internet-centered remote monitoring and controlling practices, based on the need to implement the conditions for tracking the systems online. It is witnessed that many schemes were utilized for processes such as monitoring and control. There are various critical examples in this case: PLC-SCADA based identification of faults, where protection systems are utilized to issue web-based interfaces for the users to enhance 
remote control and monitoring. Monitoring different industrial parameters centered on protocols of Zig-Bee has been applied to track temperatures, levels of water and different voltages and current ratings.

F. Seredynski in [9] has operated and various techniques have been recommended on the control and automation practices for industries using various communication initiatives and K. Lasiński in [10] is still working on how to enhance more. B. Lee in [11] projected that various communication technologies supported by two major communication media; i.e. wireless and wired, can be utilized for the transmission of data between electric utilities and smart meters. J. Bangali and A. Shaligram in [12] designed and applied cost-effective home security schemes applying GSM technologies. The scheme is considered to effectively detect burglary, hence leaks harmful gases.

S. Li, H. Sun, A. Nallanathan, L. Xu, S. Zhao and Q. Sun in [13] assessed an industrial perspective of Wireless Sensor Networks (WSNs). In the analysis, survey requirements, challenges and protocols, have concentrated on the usage of WSNs on industrial applications. Operative industrial communication has been characterized by the ideology that control and interaction has to happen in real-time, with hard time necessities.

$\mathrm{J}$. Bell in [14] conclude that there are various systems for remote control and monitoring designed as commercialized products and investigational study platforms. From the analysis, it is noted that most analysis has been done and grouped into: internet-centered monitoring servers, and GPRS modems with various approaches. GSM and SMSs protocols utilizing modules personally or combining internet technologies tracking the application of WSNs monitoring using Bluetooth, Zig-Bee and RF. Applications have varied significantly e.g. home automations, security schemes, Bio-medical applications, Bridge health monitoring, environmental monitoring and agricultural sector.

T. Vollmer and M. Manic in [15] have introduced the concept of Industrial Cyber Physical System (ICPS). In this paper, ICPS has been considered the foundational stone, which is applied in the era of industry 4.0, and in the management of eco-friendly machines. The analysis of ICPS has become a prevailing concern among many researchers, such as C. Koulamas and A. Kalogeras in [16]. ICPS incorporate physical industries and entities with cyber network to structure systems, which can operate effectively, and benefits from incorporated designs and system-wide optimizations.

\section{PROPOSED SYSTEM ARCHITECTURE}

The Industrial Cyber Physical System (ICPS) provides the basis of research among many scholars in industrial 4.0 [17]. ICPS incorporate physical entities with cyber network to structure systems, which are capable of operating with unison, and benefiting from incorporated designs and system-wide optimizations. The performance and safety of systems can be enhanced by structuring particular data infrastructures, control and monitoring approaches purposed at upholding controllability based on external disturbance and unplanned faults. With reference to the observations, deployment and design of ICPS have both practical and theoretical merits.

The similarities and differences between CPS and ICPS can be illustrated as follows: from the application perspective, ICPS is CPS, which concentrates on industrial management, control, monitoring and modelling tasks. Other than industrial based CPS, there is space initiative about CPS, which is projected at enhancing accuracy and reliability; for example, remote sensing tasks. Based on the point-of-view of scientific analysis, ICPS has to consider various human factors, because of high degree of automations, enhanced demand of operational reliability and safety of processes in industries.

Whereas most procedures can be illustrated either by exact mechanism frameworks based on physical laws or through big data enhanced realization methods based on historical information, It is evident that human factors facilitate much complexity to synthesis and analysis procedures. In that regard, modern industries, on one hand, have devoted many efforts in the structuring of automated factories, autonomous industrial robots, and structuring developed digitalized twin technologies, e.g. elimination of possible human errors, mistakes and intentional malicious threats.

On the other hand, the designs of Human in the loop and the technologies of virtual reality are utilized in dealing with tasks that include humans. Other than particular differences, there are various challenges that are shared by different forms; actual-time control and monitoring of ICPS; and incorporated plant-wide control and monitoring CPS framework. For example, it is typical that transdisciplinary cooperation is fundamental for technological transformation to happen. The enhancement of Deep Learning (DL) and $5^{\text {th }}$ generation $(5 \mathrm{G})$ wireless communication, including the establishment of novel infrastructure will significantly contribute to the designing of ICPS, its deployment that is found, and it capable of fundamentally transforming industries. ICPS is a transdisciplinary or interdisciplinary these that is still at its initial stage.

In mid 20s, the first CPSs symposium was in USA. Germany, in 2013, placed forward industry 4.0 high-technological approach to base on the ICPS research. China, in 2015, launched the 'Made in China' 2025, which is considered a ten-year approach that is meant to enhance integration of energy-based ICPSs. The European Union, China and United States have projected strategic projects for smart grids and sustainable enhancements from 2003 to 2007. Concerning smart transportation, Partner for Advancing Transportation (PATH) project was initiated in 1997 in USA. In 2010, IEEE 802.11p was released as standard and protocol for wireless communication for road vehicles.

IoE, which a concept traced by in the late $90 \mathrm{~s}$, has internet as its major source. Contrary to that, CPS networks are more heterogeneous and general. Researchers in [18] introduced the first collaborative robot in 2014 hence stimulating research and development efforts amount many scholars. It is considered that industries and governments have enhanced research on ICPSs globally. In reference to the national science foundation of America, CPSs is known as the mechanisms monitors or monitored by computer-assisted algorithms, incorporated with users and the internet. 
As for CPSs, physical and software components are intertwined each operating in various temporal and spatial scales and exhibit distinct and multiple modalities whereas interacting with each other in different ways, which transform with context. The major element of CPS is the enriching process that is has to undergo. Over the past few decades, communication and computation have been considered on the critical Cs of CPS. Implementation of the Cs depends on the design of hardware, development of software and the infrastructure of system-level task necessities,

Cognition and cloud are other elements that have attracted the attention of academia over the past few decades and thus CPS Cs are presently viewed as the major elements of this paradigm. New problems have been seen in the transdisciplinary connection necessitate by system-of-system since the platforms of big data are required to effectively manage big data included. More insights concerning Cs can be identified where self-awareness and cognition concepts are present and the hierarchical presentation tasks for ICPS optimization and monitoring are highlighted.

This research contribution concentrates on the actual-time control and monitoring aspects of ICPS. More developed approaches and possible challenges have been illustrated in the sections below. The research focusses more on the architecture for ICPS control and monitoring on a large scale, based on a particular performance controlled life cycle management system. This research segment has attracted much attention from R\&D departments, academia and industrial scholars.

\section{A. The Present Status of ICPS}

For traditional practices in industrial application, model centered methods, provide overall solutions to actual-time control and monitoring problems. These methods necessitate mathematical processing models and devices that have to be controlled, including the kind of designs that are unpractical because of the complexities stimulated by large scale distributed and hierarchical systems. For ICPS, where different control loops require monitoring, databased methods are a promising alternative meant to simplify designs. These methods are centered on data that has been retrieved from historical information, online measured signal, and therefore is considered dependent on the various physical models of the plant and the device with ICPS.

This shows that databased approaches are capable of monitoring the run-time of systems through the observable outputs of systems. The objects of monitoring are handled as black boxes or grey box schemes. It is essential to note that, even though, based on the point-of-view of data flow, databased monitoring and fault diagnosis schemes have the same underlying perspectives for the identification of the system. Targets vary and system identification is used to identify the potential faults in data instead of evaluating the undetermined parameters of the system. Heterogeneous data sources have various measurement units, calibration procedures and sampling times. As an essential data source, softened sensing methods provide actual-time evaluation of unmeasurable variables and unobserved status.

Multivariate statistical scheme monitoring methods have been utilized in various actual-time industrial domains egg automotive engineering, chemical engineering and environmental monitoring. Because of the variations in the specifications for every application, the essential response time is different from milliseconds to days e.g. in vibrant robot arms. Non-linearity, data abnormality and time-varying schemes and their potential problems have to be addressed. The factors can be considered as a major intrinsic or constraint defect in traditional techniques and the effect of performance degradation considered when traditional methods are applied to fault diagnosis and practical monitoring tasks.

With reference to this observation, various variants of the typically applied multi-variate evaluation tool has been projected to fit various actual-time requirements. It has to be considered that the core of ICPS control and monitoring system depends on the structuring of the most available actual-time data and evaluating the potential relations among interlinked systems. Nonetheless, because of the heterogeneous condition of various industrial procedures, it is fundamental to expand the scales of the considered procedures whereas applying sensor data fusion and synchronization methods.

Over the past few decades, the concept of plant-wire control and monitoring is getting much research attention from Academia and R\&D departments. This research attention includes the plant-wide control and monitoring issues that have been induced by the networked, distributed and dynamic features of the plant-wide schemes, which is considered a virtual task. How to partition and organize industrial devices and plants effectively, how to localize and detect malfunctions timely, how to design economic maintenance approaches are the most fundamental concerns that have to be managed.

Additionally, it is fundamental to structure performance evaluation methods and criteria meant to evaluate the performance degradation online, in actual-time. In this case, two fundamental dimensions for databased methods that are utilized for classification of various schemes are considered. The first one is the data structure assumption i.e. statistical models. The monitoring method based on the rule of component analysis, canonical correspondence evaluation, partial least square and independent component evaluation assure the availability of some feature space whereby the variable number is minimal compared to the real number of signals. In evaluation models, information is viewed as normally independent and distributed based on timing sequence.

To handle non-linear relationships among many variables, T. Cao in [19] projects raw information into dimensional kernel space and potentially establish regression connections in these spaces project it. In addition, a segment of linear information structures can be superimposed to exact non-linear elements. For example, Gaussian mixture models can evaluate the arbitrary distribution factor through the choice of Gaussian distributed mode with particular variances and means. According to [20], fundamental deep component analysis centered actual-time incipient fault evaluation approach has been projected where accurate operational variations and noise data in electrical drive schemes can be obtained. 
Another dimension is that of the evaluation of undetermined parameter of information structures. Scholars utilize regression analysis methods and DL in this case. For example, recursive evaluate rule component analysis that is based on regression and M. Krishnan, B. Bhowmik, A. Tiwari and B. Hazra in [21] have projected fault identification method for slow time-variant procedures. This was done though online monitoring of the variations of regression connections that is adapted to novel factors and conditions. With the projected remedies, based on first order perturbation, it is potential to update test statistics that can match the updated regression relationships between system input and output for every novel online measurement.

For learning-centered methods, K. Bung in [22] has projected the locality learning algorithms, weighted projections and regression (WPR), which has been incorporated wit component analysis, and enhanced component analysis for non-linear fault identification. WPR identifies the reception fields for every localized structure of data that refers to the case from linear functional family, which is meant to represent the responsible input space. The overall number of localized structure of data is adjusted based on learning from information, and the reception segments that correspond to every local structure of data. Incorporate designs of process control and fault diagnosis scheme across hardware application and software implementation levels is considerably advantageous.

The integrated design methods are consistent with reference to the CPS concepts, where physical entities of software and hardware, including network set-ups have been correlated to virtual algorithms that have been optimized based on the same system. Application-particular processing platforms have been structured for actual-time monitoring aims. For example, a play-and-plug module for monitoring processes applied in multi-core processors has been evaluated in [23]. Dynamic computational capacities and effective smart algorithms are instrumental in new actual-time ICPS remedies. Over the past few decades, leading communication and automotive industries have been structuring particular hardware for DL computation, which is known as Neural Processing Units (NPUs). A significant problem for the embedded schemes is to identify optimal balance between the consumption of power and its performance. NPUs are centered on databased parallel computational architecture, mostly effective at evaluating big data such as images and videos. In this case, time control and monitoring of ICPSs is considered.

Industrial control and monitoring of eco-friendly machines is an interlinkage of algorithms, mechanisms and architectures utilized in the industrial segment for control and monitoring activities of industrial procedures, devices, machines, motors applied in company premises to accomplish particular goals. Irrespective of the fact that is sounds good to incorporate industrial ecosystem in the near future, there are many problems to be encountered due to big data and the communication of data throughout various platforms. The current project has also touched on the industrial application, which will be monitored through sensor sets constituting sensor modules. These modules gather essential information meant to determine whether application have to be monitored and if they are operational under particular threshold values.

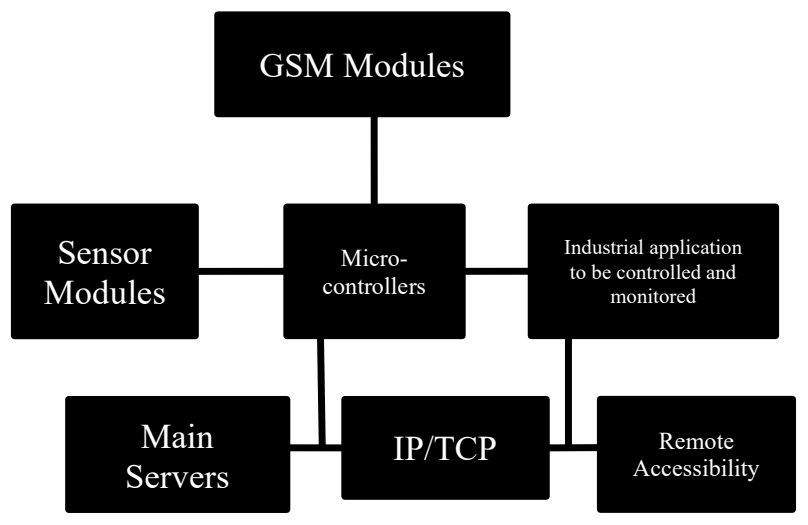

Fig 2: Projected system architecture

Information from different sensors in sensor modules is computed to the controlling device majorly the microcontroller. The controlling devices have been placed into interfaces with GPRS-based GSM module that can be accessed remotely by different users. The control devices are capable of simultaneously forwarding information to the main servers. The main servers found at the company premise highlight corresponding information that has been received from the various controlling devices. The arrangements of reaching the main serves remotely via mobile devices can be attained through IP and TCP protocols; therefore, the control and monitoring of application can be accomplished through remote accessibility.

In case the industrial application is not operational after being controlled or monitored, remote control by users of mobile can be done through the accessibility of controllers using GPRS-based GSM modules. Communication through GPRS provides continuous, cheap and secure communication to users where internet accessibility is not guaranteed. Therefore, industrial application will be controlled and monitored through GPRS communication methods without any potential restrictions to infrastructure or distance. It is therefore significant to follow and refer to the comparisons of the various communication methods as shown in Table 1 below. 
Table 1: Various comparisons of communication methods

\begin{tabular}{|c|c|c|c|c|c|}
\hline Features & Zig-Bee & GPRS-GSM & WiFi & Bluetooth & RF \\
\hline Focal area & $\begin{array}{l}\text { Control and } \\
\text { Monitoring }\end{array}$ & $\begin{array}{l}\text { Wide-rage sound and } \\
\text { information transfromatio } \\
\text { control and monitoring }\end{array}$ & $\begin{array}{l}\text { Email, Web } \\
\text { and image }\end{array}$ & Other than cable & Wireless service \\
\hline System resources & $32 \mathrm{~kb}$ & $16+\mathrm{mb}$ & $1+\mathrm{mb}$ & $250+\mathrm{kb}$ & $32 \mathrm{~kb}$ \\
\hline $\begin{array}{c}\text { Networking } \\
\text { information width }\end{array}$ & 20 to 250 & 64 to $128+$ & $1,100+$ & 720 & 32 \\
\hline Coverage segment & 1 to 100 & 1 to $100+$ & 1 to 100 & 1 to $100+$ & 1 to $100+$ \\
\hline Performance segment & $\begin{array}{l}\text { Endurance expense } \\
\text { energy consumption }\end{array}$ & Accessibility quality & $\begin{array}{l}\text { Velocity } \\
\text { resilience }\end{array}$ & Expense comfort & Accessiblity \\
\hline
\end{tabular}

\section{B. Wireless Communication}

The concept of wireless communication denotes to the transfer of information thorough distances without applying electrical wires or conductors. The distance included may be less as in the TV remote control or more distances as in radio communication. This incorporates various forms of portable, mobile and fixed cellular telephones, Personized Digital Assistant (PDA), and wireless networks. In this paper, we utilize the Zig-Bee network for industrial information communication.

\section{1) Zig-Bee Rule}

Zig-Bee is considered a wireless network rule or protocol particularly structured from minimal data rate sensor and control network. Zig-Bee applies IEEE 802.15.40 MAC layers and physical layers to issue standard-centered, reliable wireless information transfer (Fig 3). The applications using this protocol include architectural automation network, security schemes, patient monitoring, remote metering and company control network among others. Zig-Bee utilizes DSSS signals of radio in $2.40 \mathrm{GHs}-\mathrm{ISM}$ bands available globally, $915 \mathrm{MHz}$ bands for N. America and $868 \mathrm{MHz}$ band for Europe.

For the 2.40 GHs-ISM bands, there are 16 channels that have been defined, everyone has three channels, and $\mathrm{MHz}$ based on $5 \mathrm{MHz}$, providing $2 \mathrm{MHz}$ gap between the different channel pairs. The protocol utilizes eleven-chip pin code with found data bits that have been encoded into individual symbol. It will provide maximum rate of data of $129 \mathrm{Kbps}$. The MAC layers and physical layers have been considered in IEEE 802.15.40. The operational groups share various design features as IEEE $802.11 \mathrm{~b}$ protocol.

$$
\text { Features of Zig-Bee Rule }
$$

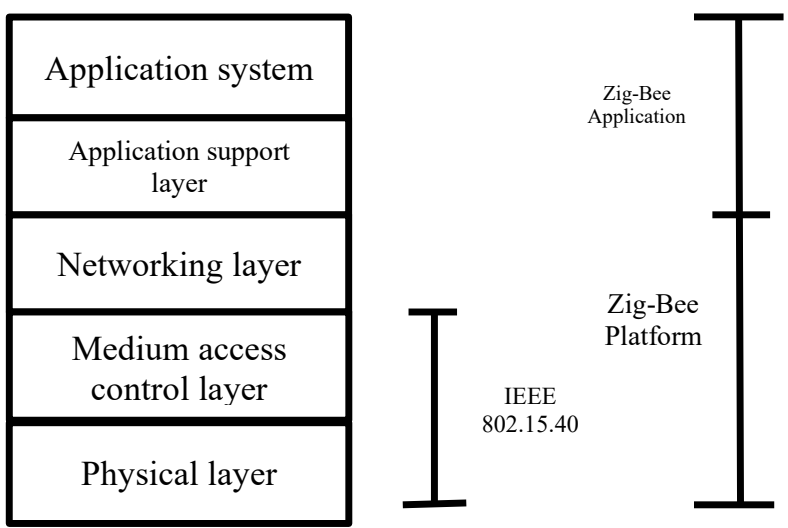

Fig 3: Stack and layers of the Zig-Bee protocol

Zig-Bee has minimal duty cycles that provide longevity in the life of batteries. AES that is 128-bit of encryption gives secure connection between the different devices. The protocol is capable of supporting collision elimination ad more than 64000 network nodes. The protocol supports mesh network, dynamic network and static network topologies.

The protocol does not transform the channel in instances of heavier interference. However, it depends on its low duty cycles and collision-elimination algorithm to reduce the loss of data due to collision effects. The protocol specifies the 
collision-avoidance algorithms, which are the same as the IEEE 802.11b and every device is capable of listening to the different channels before transferring to potentially limit collision frequency between the various Zig-Bee devices.

4) GSM Networks

GSM is internationally acknowledged protocol for digitalized cellular communication. The digitalized condition of GSM permits the transfer of information (i.e. asynchronous and synchronous) to ISDN terminal; however, the most major telephony service is entirely supported by GSM. The novel segment of GSM is Short Message Services (SMSs), and this represents the bi-direction services used to communicate short messages that are alphanumerical in nature in its forwardstore process.

\section{System's Hardware}

For control and monitoring to be attained in industrial application, certain factors have to be considered in the designing of the system:

\section{1) Micro-Controllers}

Micro-controllers projected for the system design is AT-Mega 32A that represents the low-energy CMOS-8-bit microcontroller centered on AVR-RISC architecture. Through the execution of the detailed instructions in the same clock cycles, AT-Mega 32A attains its throughput that is about one MIPS/MHz permitting optimal optimization of energy use against the speeds of processing. Handling numerous tasks in this projected system involves the controller to effectively execute instructions momently and timely. Therefore, AT-Mega 32A is effective for the process of computation with effective programmable flashing memory of about $32 \mathrm{~kb}$.

\section{2) Current Sensors}

Allegro-ACS-712 gives economical and accurate remedies for DC and AC current sensing in communication, commercial and industrial systems. The packaged device permits for easier application. Normal application of the sensors is for monitoring motors and their current fault' protection. Device output incorporate positive (+ve) slope i.e. ( $>+$ ve-OUT) whenever an increased flow of current through primary copper conduction paths including pin 1, pin 2 , pin 3 and pin 4 is considered the path for current sampling (see Fig 4).

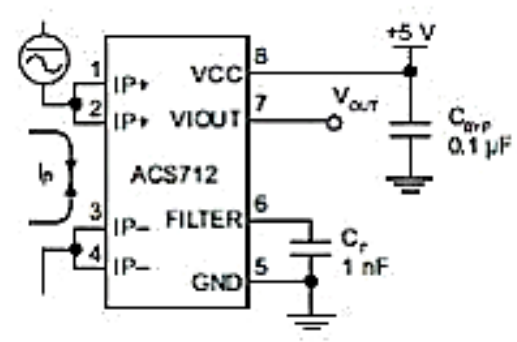

Fig 4: Plan of Current sensor IC

The resistance in the interior part of the conductive paths is $1.2 \mathrm{~m} \Omega$ and issues low losses of power. Copper conductors' thickness permits the survival of devices up to five times the over-current condition. Terminals of the conductive paths are isolated from the leads of signals (see Fig 4 for pin 5 and pin 8). This permits ACS -712 to be utilized to monitor currents and safeguarding the motor based on the current factors in the industry.

3) Temperature Sensors

$L M-35$ Series are considered as incorporated circuit temperature sensors with voltage outputs proportional to centigrade or Celsius temperature (see Fig 5). The series therefore are advantageous over the linear temperatures and sensors, which have been calibrated to Kelvin, as the users are not supposed to deduct significant voltages from outputs to retrieve centigrade scale. The series' low output impedance, precise inherent calibration and linear outputs make interfacing to the control and readout circuitry easier to underpin.

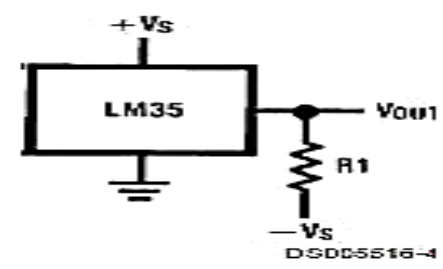

Fig 5: Representation of the LM-35 series

The series would be utilized as a circuit or sensor of temperatures. These can be based on temperatures and the surface. It is considered that ambient temperature is just like the surface temperature. Series sensor temperature of $R_{1}=-V S$ per 
$50 \mu \mathrm{A}$ and the voltages evaluated for the correspondent sensed temperatures $-V O U T=+1300 \mathrm{mV} @+130^{\circ} \mathrm{C}=$ $+230 \mathrm{mV} @+23^{\circ} \mathrm{C}=-530 \mathrm{mV} a t-53^{\circ} \mathrm{C}$.

4)

GSM Modules

TC-35-i GSM modules operate in GSM-900 MHz and GSM-1800 MHz bands of frequencies. These are completely slim and compact communication modules that have been structured for telephony, telematics, and telemetry. It provides more features e.g. AT commands for industrial ecosystem and SIM application toolkit. The physical layer and interface to cellular application is structured using ZIF connectors. It incorporates 40 pins essential for the process of controlling units, communicating information and audio signal and generating lines of power.

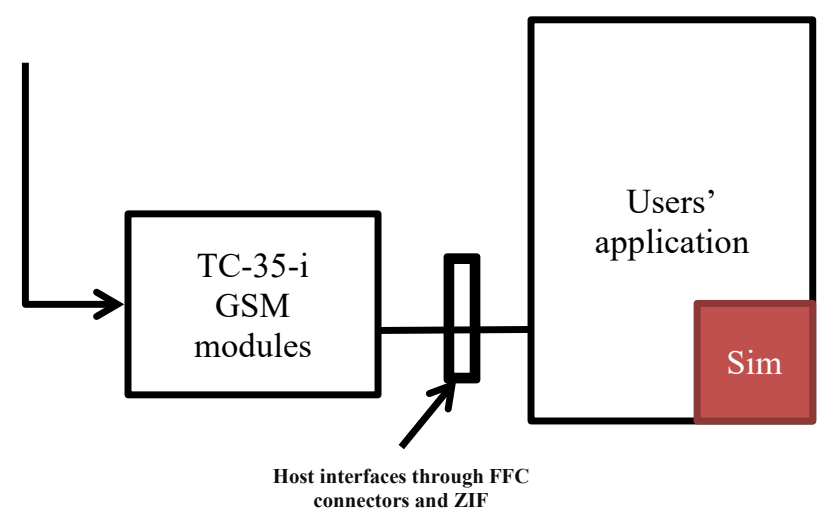

Fig 6: User application of the TC-35-i GSM modules

The modules are applied (see Fig 6) in battery - power industrial application. The cellular device is applied in Man Machine Interfaces (MMIs), which represents a series of interfaces allowing the accessibility of the module. In the batterpower application, TC-35-i elements of charging controls can be utilized for the Li-ion batteries. The module aids in the tracking of system locations applied in industries for users at various locations purposing to communicate with systems. In that regard, industrial applications and parameters more than its projected threshold figure can be controlled through microcontrollers based on TC-35-I GSM. As for the cellular application, in an aim to make them operate effectively, it is fundamental that the modules are securely connected to the host.

\section{CONCLUSION AND FUTURE DIRECTION}

In conclusion, this research contribution has projected a design and application of IoE for controlling and monitoring different parameters and applications in industries based on GPRS wireless communication methods. The main idea in this contribution is to issue connectivity and longevity between industrial users and the environment. The main merit of the developed scheme is to incorporate progressive monitoring on various industrial applications to correctly control them over different threshold setting. This system has incorporated features such as sensor modules, micro-controllers, main service, and GSM module. However, to guarantee longevity and connectivity, future research should concentrate on adding more features to the propped system. The main rationale of the future research should be directed to establishing reliable intelligence industrial control and monitoring systems using WSNs.

\section{References}

[1]. S. Alexander and T. Gor, "Monitoring, diagnosis and control of industrial processes", Computers \& Industrial Engineering, vol. 35, no. 1-2, pp. 193-196, 1998. Available: 10.1016/s0360-8352(98)00059-x.

[2]. M. Mansouri, "Optimal sensor and path selection for target tracking in wireless sensor networks", Wireless Communications and Mobile Computing, vol. 14, no. 1, pp. 128-144, 2012. Available: $10.1002 / \mathrm{wcm} .1241$.

[3]. M. You and T. Akashi, "Multi-view face detection using frontal face detector", IEEJ Transactions on Electrical and Electronic Engineering, vol. 13, no. 7, pp. 1011-1019, 2018. Available: 10.1002/tee.22658.

[4]. G. V. Iyer, "Eco-Friendly Rubberized Cotton Fabric Roller for Ginning Machines", Journal of Agricultural Safety and Health, vol. 13, no. 1, pp. 33-43, 2007. Available: 10.13031/2013.22310.

[5]. G. Petrilli, "Implications of environmental measures for industrial development Community ecological policy: Consequences of action to improve environment on industrial development", Science of The Total Environment, vol. 1, no. 4, pp. 323-340, 1973. Available: 10.1016/00489697(73)90023-5.

[6]. A. Ogden-Swift, "Applying New Control Technologies to Improve Process Profit", Measurement and Control, vol. 29, no. 8, pp. 233-236, 1996. Available: $10.1177 / 002029409602900803$.

[7]. X. Xiaoli and W. Huan, "The Wireless Sensor Network Construction of the Photovoltaic Power System Zig Bee", Procedia Engineering, vol. 15, pp. 2511-2515, 2011. Available: 10.1016/j.proeng.2011.08.472.

[8]. A. Çakir, H. Çalis and G. Turan, "Remote Controlling and Monitoring of Induction Motors Through Internet", TELKOMNIKA Indonesian Journal of Electrical Engineering, vol. 12, no. 12, 2014. Available: 10.11591/telkomnika.v12i12.6719.

[9]. F. Seredynski, "Control and automation of rolling processes in steel and nonferrous industries", Metals Technology, vol. 2, no. 1, pp. 486-493, 1975. Available: 10.1179/030716975803277032. 
[10]. K. Łasiński, "Cam_Gate System in Product Quality Control, Optimal Selection of Inspecting-Sorting Automatic Machines", Solid State Phenomena, vol. 198, pp. 445-450, 2013. Available: 10.4028/www.scientific.net/ssp.198.445.

[11]. B. Lee, "Reviewer Acknowledgements for Network and Communication Technologies Vol. 4, No. 1", Network and Communication Technologies, vol. 4, no. 1, p. 52, 2019. Available: 10.5539/nct.v4n1p52.

[12]. J. Bangali and A. Shaligram, "Design and Implementation of Security Systems for Smart Home based on GSM technology", International Journal of Smart Home, vol. 7, no. 6, pp. 201-208, 2013. Available: 10.14257/ijsh.2013.7.6.19.

[13]. S. Li, H. Sun, A. Nallanathan, L. Xu, S. Zhao and Q. Sun, "Industrial Wireless Sensor Networks", International Journal of Distributed Sensor Networks, vol. 10, no. 8, p. 218050, 2014. Available: 10.1155/2014/218050.

[14]. J. Bell, "Remote Monitoring and Control of Water Management Systems, via Satellite", Measurement and Control, vol. 20, no. 5, pp. 81-87, 1987. Available: $10.1177 / 002029408702000503$.

[15]. T. Vollmer and M. Manic, "Cyber-Physical System Security With Deceptive Virtual Hosts for Industrial Control Networks", IEEE Transactions on Industrial Informatics, vol. 10, no. 2, pp. 1337-1347, 2014. Available: 10.1109/tii.2014.2304633.

[16]. C. Koulamas and A. Kalogeras, "Cyber-Physical Systems and Digital Twins in the Industrial Internet of Things [Cyber-Physical Systems]", Computer, vol. 51, no. 11, pp. 95-98, 2018. Available: 10.1109/mc.2018.2876181.

[17]. O. Kapitonov, "Cyber-physical Systems in electrochemical measurements", System technologies, vol. 4, no. 129, pp. 3-7, 2020. Available: 10.34185/1562-9945-4-129-2020-01.

[18]. R. KURAZUME, "An example of IOT for service robot system", The Proceedings of Mechanical Engineering Congress, Japan, vol. 2016, no. 0, p. F161005, 2016. Available: 10.1299/jsmemecj.2016.f161005.

[19]. T. Cao, "A Combined Dimensional Kernel Method for Graph Classification", Journal of Information Technology Research, vol. 10, no. 3, pp. 2233, 2017. Available: 10.4018/jitr.2017070102.

[20]. "Optimal use of principal component analysis", Deep Sea Research Part B. Oceanographic Literature Review, vol. 34, no. 12, p. $1078,1987$. Available: 10.1016/0198-0254(87)96149-8.

[21]. M. Krishnan, B. Bhowmik, A. Tiwari and B. Hazra, "Online damage detection using recursive principal component analysis and recursive condition indicators", Smart Materials and Structures, vol. 26, no. 8, p. 085017, 2017. Available: 10.1088/1361-665x/aa7220.

[22]. K. Bung, "Teaching Algorithms and Learning Algorithms", Programmed Learning and Educational Technology, vol. 19, no. 3, pp. 181-218, 1982. Available: $10.1080 / 0033039820190302$.

[23]. V. H.R, D. K and D. S, "A Plug and Play Module to identify type of Modulation", International Journal of Engineering and Technology, vol. 9, no. 4, pp. 3130-3137, 2017. Available: 10.21817/ijet/2017/v9i4/170904062. 